\title{
Erratum
}

European Journal of Clinical Nutrition (2000), 54, 195-202

\section{Total daily energy expenditure and pattern of physical activity measured by minute-by-minute heart rate monitoring in 14-15 year old Swedish adolesce'nts}

\author{
U Ekelund, M Sjöström, A Yngve and A Nilsson \\ Unfortunately due to a typesetting error Tables 4 and 5 were reproduced incorrectly. \\ The correct versions are printed below:
}

Table 4 Absolute time (min/day) and relative time (\%) spent at different levels of physical activity assessed by heart rate monitoring (mean \pm s.d.)

\begin{tabular}{lccccc}
\hline Gender $(n)$ & Min registered & $>$ HR flex & $\geq 4.5$ SEE & $\geq 50 \% \mathrm{PVO}_{2}$ & $\geq 70 \% \mathrm{PVO}_{2}$ \\
\hline Boys (42) & $788 \pm 71$ & $233 \pm 147$ & $67 \pm 71$ & $26 \pm 17$ & $7 \pm 7$ \\
(range) & $(642-954)$ & $(63-514)$ & $(4-155)$ & $(2-71)$ & $(0-24)$ \\
Percentage of reg time & 100 & $30 \pm 18$ & $8.6 \pm 9.2$ & $3.3 \pm 2.2$ & $1.0 \pm 0.8$ \\
Girls (40) & $793 \pm 75$ & $220 \pm 130$ & $67 \pm 76$ & $32 \pm 27$ & $10 \pm 12$ \\
(range) & $(665-924)$ & $(48-526)$ & $(2-334)$ & $(2-90)$ & $(0-39)$ \\
Percentage of reg time & 100 & $28 \pm 15$ & $8.6 \pm 9.8$ & $4.0 \pm 3.5$ & $1.3 \pm 1.5$ \\
\hline
\end{tabular}

HR Flex, average of the highest HR during resting measurements and lowest $\mathrm{HR}$ during exercising; $\geq 4.5 \mathrm{SEE}, \mathrm{HR}$ corresponding to 4.5 times $\mathrm{SEE} ; \geq 50 \% \mathrm{PVO}_{2}$, $\mathrm{HR}$ corresponding to $50 \% \mathrm{PVO}_{2} ; \geq 70 \% \mathrm{PVO}_{2}, \mathrm{HR}$ corresponding to $70 \%$ $\mathrm{PVO}_{2}$.

Table 5 Comparison of energy expenditures and activity levels between minute-by-minute heart rate monitoring studies in $14-15 \mathrm{y}$ old adolescents

\begin{tabular}{lcccc}
\hline & $\begin{array}{c}\text { Spurr \& } \\
\text { Reina }(1988)^{\mathrm{a}}\end{array}$ & $\begin{array}{c}\text { Spurr \& } \\
\text { Reina }(1988)^{\mathrm{b}}\end{array}$ & $\begin{array}{c}\text { Livingstone } \\
\text { et }\end{array}$ & Present \\
(1992) & study \\
\hline Boys & & & & \\
Subjects (n) & 20 & 26 & 3 & 42 \\
Registered time (days) & $1-2$ & $1-2$ & $2-3$ & $2-3$ \\
Age (y) & 14.8 & 14.8 & 15.4 & 14.8 \\
Weight (kg) & 49.9 & 38.9 & 50.7 & 61.6 \\
TDEE (MJ/day) & 12.1 & 10.6 & 11.5 & 12.8 \\
TDEE (kJ/kg/day) & 244 & 274 & 227 & 210 \\
AEE (MJ/day) & $5.6^{\mathrm{c}}$ & $4.9^{\mathrm{c}}$ & 4.8 & 5.4 \\
PAL & 1.94 & 1.93 & 1.71 & 1.74 \\
Girls & & & & \\
Subjects (n) & 19 & 22 & 3 & 40 \\
Registered time (days) & $1-2$ & $1-2$ & $2-3$ & $2-3$ \\
Age (y) & 14.9 & 15.2 & 15.6 & 14.7 \\
Weight (kg) & 49.3 & 42.0 & 55.4 & 55.9 \\
TDEE (MJ/day) & 8.3 & 8.2 & 9.9 & 10.0 \\
TDEE (kJ/kg/day) & 174 & 203 & 179 & 182 \\
AEE (MJ/day) & $2.7^{\mathrm{cc}}$ & $2.8^{\mathrm{cc}}$ & 4.4 & 4.0 \\
PAL & 1.61 & 1.61 & 1.88 & 1.67 \\
\hline
\end{tabular}

${ }^{\mathrm{a} C}$ Control subjects; ${ }^{\mathrm{b}}$ Marginally malnourished subjects; ${ }^{\mathrm{c}}$ Values have been calculated by the present authors.

AEE, activity energy expenditure (TDEE - BMR); PAL, physical activity level (TDEE/BMR).

We apologise for any inconvenience this may have caused. 